\title{
CMP-N-acetylneuraminic acid synthetase interacts with fragile $X$ related protein 1
}

\author{
YUN MA $^{1,2}$, SHUAI TIAN $^{1}$, ZONGBAO WANG $^{2}$, CHANGBO WANG $^{1}$, \\ XIAOWEI CHEN ${ }^{1}$, WEI LI ${ }^{1}$, YANG YANG ${ }^{1}$ and SHUYA HE ${ }^{1,2}$ \\ ${ }^{1}$ Department of Biochemistry \& Biology; ${ }^{2}$ Hunan Province Cooperative Innovation Center for Molecular Target New Drug Study, \\ Institute of Pharmaceutical and Biological Sciences, University of South China, Hengyang, Hunan 421001, P.R. China
}

Received June 12, 2015; Accepted May 25, 2016

DOI: $10.3892 / \mathrm{mmr} .2016 .5438$

\begin{abstract}
Fragile X mental retardation protein (FMRP), fragile $\mathrm{X}$ related 1 protein (FXR1P) and FXR2P are the members of the FMR protein family. These proteins contain two KH domains and a RGG box, which are characteristic of RNA binding proteins. The absence of FMRP, causes fragile X syndrome (FXS), the leading cause of hereditary mental retardation. FXR1P is expressed throughout the body and important for normal muscle development, and its absence causes cardiac abnormality. To investigate the functions of FXR1P, a screen was performed to identify FXR1P-interacting proteins and determine the biological effect of the interaction. The current study identified CMP-N-acetylneuraminic acid synthetase (CMAS) as an interacting protein using the yeast two-hybrid system, and the interaction between FXR1P and CMAS was validated in yeast using a $\beta$-galactosidase assay and growth studies with selective media. Furthermore, co-immunoprecipitation was used to analyze the FXR1P/CMAS association and immunofluorescence microscopy was performed to detect expression and intracellular localization of the proteins. The results of the current study indicated that FXR1P and CMAS interact, and colocalize in the cytoplasm and the nucleus of HEK293T and HeLa cells. Accordingly, a fragile X related 1 (FXRI) gene overexpression vector was constructed to investigate the effect of $F X R 1$ overexpression on the level of monosialotetrahexosylganglioside 1 (GM1). The results of the current study suggested that FXR1P is a tissue-specific regulator of GM1 levels in SH-SY5Y cells, but not in HEK293T cells. Taken together, the results initially indicate that FXR1P interacts with CMAS, and that FXR1P may enhance the activation of sialic acid via interaction with CMAS, and increase GM1 levels to affect the
\end{abstract}

Correspondence to: Professor Yun Ma or Professor Shuya He, Department of Biochemistry \& Biology, University of South China, 28 Western Changshen Road, Hengyang, Hunan 421001, P.R. China E-mail: luckymayun@163.com

E-mail: skyhe2000@hotmail.com

Key words: fragile $\mathrm{X}$ related protein 1, CMP-N-acetylneuraminic acid synthetase, fragile $\mathrm{X}$ syndrome, GM1 development of the nervous system, thus providing evidence for further research into the pathogenesis of FXS.

\section{Introduction}

Fragile X syndrome (FXS) is the most common inherited developmental disorder. The disorder results from defects in the fragile X mental retardation 1 (FMRI) gene, and lack of FMRI expression caused by hyper-expansion and methylation of CGG repeats in the first exon of this gene (1-3). The FMR protein family has three highly homologous members. The fragile $\mathrm{X}$ mental retardation protein (FMRP) is coded by the FMRI gene. Two other members are the fragile $\mathrm{X}$ related protein 1 (FXR1P) (4) and FXR2P (5), which are coded by the fragile $\mathrm{X}$ related 1 ( $F X R 1$ ) gene and $F X R 2$, respectively. These proteins possess two KH domains and a RGG box, which suggests that they are involved in intracellular RNA transport and translation regulation (6-10). They also contain a nuclear localization signal and a nuclear export signal, which suggests that they can shuttle between the nucleus and the cytoplasm (11).

FMRP exhibits similar sequence identity $(>60 \%)$ to FXR1P/FXR2P, and also contains two KH domains and RGG box (12). The absence of FMRP is the cause of FXS, however, it is not established whether FXR1P and FXR2P are associated with FXS pathology or phenotype. Additionally, it is not established whether FXR1P and FXR2P may compensate for the absence of FMRP. Furthermore, no significant neuropathological abnormalities were demonstrated in fetal brain tissue from FXS with FXR1P and FXR2P are normally expressed (13). FMRP, FXR1P and FXR2P can interact with themselves and each other $(5,14,15)$, however, their distributions demonstrated individual expression patterns in certain mouse and human tissues $(16,17)$, which indicates that each protein also may function autonomously (18).

FXR1P is expressed throughout the body, particularly in muscle and heart tissue. There are seven FXR1P isoforms in mouse $(19,20)$, including three muscle-specific isoforms (20) and one cardiac-specific isoform (21). FXR1P normal expression is essential for postnatal viability. A previous study demonstrated that inactivation of FXR1P in mice caused the death of neonates shortly after birth, which was likely due to abnormal development of the myocardiac or respiratory muscle (20). In Xenopus, FXR1P regulates the development 
of somites, eyes and cranial cartilage, however, reduction of FXR1P disrupted the rotation and segmentation of somitic myotomal cells and hindered normal myogenesis (22). In a previous study in zebrafish, inactivation of FXR1P caused abnormalities in striated muscle and severe cardiomyopathy, and led to heart failure in embryos (23). In humans, altered expression of muscle-specific isoforms of FXR1P has been previously implicated in facioscapulohumeral muscular dystrophy (24). Additionally, a previous study demonstrated that inactivation of FXR1P enhanced the expression level of tumor necrosis factor- $\alpha$ in mouse macrophages (25). Collectively, these previous studies demonstrated that FXR1P has an important regulatory function in the development of normal and cardiac muscle $(26,27)$.

\section{Materials and methods}

Strains and cell lines. The Escherichia coli (E.coli) strains, DH5 $\alpha$ and TOP10 (China Center for Type Culture Collection, Wuhan, China), were cultured in LB medium (1\% tryptone, $0.5 \%$ yeast extract, $1 \% \mathrm{NaCl}, \mathrm{pH} 7.0$; Oxoid; Thermo Fisher Scientific, Inc., Waltham, MA, USA) at $37^{\circ} \mathrm{C}$. The Saccharomyces cerevisiae strains, Y187 and AH109, were cultured at $30^{\circ} \mathrm{C}$ in yeast extract peptone dextrose (YPD) medium $(2 \%$ tryptone, $1 \%$ yeast extract, and $2 \%$ glucose; Oxoid; Thermo Fisher Scientific, Inc.) or SC medium ( $0.67 \%$ yeast nitrogen base without amino acids, $2 \%$ glucose) supplemented with an amino acid mixture without the indicated amino acids for selection. HeLa and HEK293T cells (Academy of Military Medical Sciences, Beijing, China) were cultured in Dulbecco's modified Eagle's medium (DMEM; Gibco; Thermo Fisher Scientific, Inc.) supplemented with $10 \%$ fetal calf serum (Zhejiang Tianhang Biotechnology Co., Ltd., Hangzhou, China) in a humidified $5 \% \mathrm{CO}_{2}$ incubator at $37^{\circ} \mathrm{C}$. SH-SY5Y cells were cultured in DMEM:nutrient mixture F12 supplemented with $10 \%$ fetal calf serum in a humidified $5 \% \mathrm{CO}_{2}$ incubator at $37^{\circ} \mathrm{C}$.

Plasmids. For two-hybrid screening, the full open reading frame of the human FXRl (GenBank NM_005087.3) gene was subcloned into the pGBKT7 vector (28) (Clontech Laboratories, Inc., Mountainview, CA, USA) to construct pGBKT7-FXR1 (Table I), which was used as the bait plasmid. In the co-immunoprecipitation assay, the full open reading frames of the human FXRl gene and human CMP-N-acetylneuraminic acid synthetase (CMAS) gene (GenBank NM_018686.4) were subcloned into the pCMV-HA vector (28) (Clontech Laboratories, Inc.) and pCMV-Myc vector (28) (Clontech Laboratories, Inc.) to construct the recombinant vectors, pCMV-HA- $F X R 1$ and pCMV-Myc-CMAS. In the colocalization assay, the full open reading frame of the human FXRl gene and human CMAS gene were subcloned into the pEGFP-N1 vector (28) (Clontech Laboratories, Inc.) and pDsRed-Monomer-N1 vector (28) (Clontech Laboratories, Inc.), respectively, to construct pEGFP-N1-FXRl and pDsRed-Monomer-N1-CMAS (Table I). Additionally, the full open reading frame of the human $F X R l$ gene was inserted into the pcDNA3.1(-) vector (29) (Clontech Laboratories, Inc.) to generate the recombinant vector, pcDNA3.1(-)-FXRI (Table I), which was transfected into SH-SY5Y cells using Lipofectamine 2000 (Invitrogen; Thermo Fisher Scientific,
Inc.) and biological effect of the interaction between FXR1P and CMAS was detected.

Yeast two-hybrid screening. All procedures were performed out according to the yeast two-hybrid system manufacturer's instructions (Clontech Laboratories, Inc.). The yeast strain AH109 (Clontech Laboratories, Inc.) was first transformed with the pGBKT7-FXR1 recombinant plasmid, which was used as the bait to screen the library. The Human Fetal Brain Matchmaker cDNA library (Clontech Laboratories, Inc.) cloned into the vector pGADT7 was transformed into the yeast strain Y187, which was used as prey for two-hybrid screening (30). The yeast strain AH109-pGBKT7-FXR1 was mated with Y187 pre-transformed with the cDNA library and then cultured for $20 \mathrm{~h}$ in YPD adenine medium supplemented with kanamycin to produce diploid zygotes. The products were plated on leucine, tryptophan and histidine-free SD medium $(\mathrm{SD} / \mathrm{LTH})$ and incubated for 3 days at $30^{\circ} \mathrm{C}$. After 3 days, colonies on individual $\mathrm{SD} / \mathrm{LTH}$ plates were counted and the total number of transformants was calculated. To verify putative interactions between bait protein and prey proteins from the cDNA libraries, the colonies present on SD/LTH medium were collected and assayed for $\beta$-galactosidase ( $\beta$-gal) activity (31). The $\beta$-gal activity assay was conducted using a colony-lift filter assay according to the manufacturer's instructions (Clontech Laboratories, Inc.). Subsequently, the library from the positive colonies (positive growth in SD/LTH media and positive $\beta$-gal activity) were extracted and digested with Hind III using a Qiagen Plasmid Midi and Maxi kit (Qiagen $\mathrm{GmbH}$, Hilden, Germany) according to the manufacturer's instructions. Plasmid fragment sizes were analyzed by $1 \%$ agarose gel electrophoresis to segregate colonies. Plasmid DNA purified from the positive yeast colonies was used to transform E.coli TOP10. Finally, the identity of the positive clones was determined by sequencing and Basic Local Alignment Search Tool searches.

Validation of the interaction between CMAS and FXRI using the yeast two-hybrid system. The purified bait plasmid pGBKT7-FXRl was transformed into the AH109 strain and was then cultured on tryptophan-free SD medium (SD/T) plates for toxicity and autonomous activation assays (32). The recombinant vector pGADT7-CMAS was transformed into the yeast strain Y187 and then plated on SD medium lacking leucine (SD/L) (33) (Oxoid; Thermo Fisher Scientific, Inc.). Subsequently, AH109-pGBKT7-FXR1 was mated to Y187-pGADT7-CMAS. The transformants were cultured in yeast peptone dextrose adenine medium for $20 \mathrm{~h}$ and then plated on the SD/LTH. The interaction between CMAS and FXR1P was confirmed following colony formation on the plates and $\beta$-gal assays.

Co-immunoprecipitation assay. HEK293T cells were maintained in DMEM medium supplemented with $10 \%$ fetal bovine serum (FBS), $100 \mathrm{U} / \mathrm{ml}$ penicillin and $100 \mathrm{mg} / \mathrm{ml}$ streptomycin. At 70\% confluency, HEK293T cells were seeded into 6-well plates with basal serum and antibiotic-free DMEM. According to manufacturer's protocols, the plasmids pCMV-HA- $F X R 1$ and pCMV-Myc-CMAS were co-transfected into HEK293 cells with Lipofectamine 2000 (Invitrogen; 
Table I. Plasmids used in the current study.

\begin{tabular}{|c|c|c|c|c|}
\hline Plasmid name & Vector & Insert(s) & Insertion site & Primers (5'-3') \\
\hline pGBKT7-FXR1 & pGBKT7 & FXR1 & $E c o R$ I & $\begin{array}{c}\text { ctagaattcatggcggacgtgacggtgctagaatt } \\
\text { cttatgaaacaccattc }\end{array}$ \\
\hline pCMV-HA-FXR1 & pCMV-HA & FXR1 & EcoR I, XhoI & $\begin{array}{c}\text { tatgaattcggatggcggagctgacggtggaggcg } \\
\text { ctcgagttatgaaacaccattcaggac }\end{array}$ \\
\hline pCMV-Myc-CMAS & pCMV-Myc & BTF & EcoR I, XhoI & $\begin{array}{c}\text { gtagaattccgatggactcggtggagcgtctcgag } \\
\text { ctatttttggcatgaat }\end{array}$ \\
\hline pEGFP-N1-FXR1 & pEGFP-N1 & FXR1 & Xho I, EcoR I & $\begin{array}{c}\text { tatctcgagctatggcggagctgacggtggaggcg } \\
\text { gaattcttatgaaacaccattcaggac }\end{array}$ \\
\hline $\begin{array}{l}\text { pDsRed-Monomer- } \\
\text { N1-CMAS }\end{array}$ & $\begin{array}{l}\text { pDsRed- } \\
\text { Monomer }\end{array}$ & BTF & Xho I, EcoR I & $\begin{array}{c}\text { tatctcgagctatggactcggtggagggcgaattc } \\
\text { ctatttttggcatgaat }\end{array}$ \\
\hline pcDNA3.1(-)-FXR1 & pcDNA3.1(-) & FXR1 & EcoR I, XhoI & $\begin{array}{c}\text { tatctcgagctatggcggagctgacggtggaggcg } \\
\text { gaattctatgaaacaccattcaggac }\end{array}$ \\
\hline
\end{tabular}

Thermo Fisher Scientific, Inc.), and pCMV-HA-FXRl and pCMV-Myc-CMAS were transfected as controls. After $5 \mathrm{~h}$, the cells were cultured with DMEM containing 10\% FBS, $100 \mathrm{U} / \mathrm{ml}$ penicillin and $100 \mathrm{mg} / \mathrm{ml}$ streptomycin. After $48 \mathrm{~h}$, the transfected cells were collected, and then lysed with RIPA lysis buffer containing protease inhibitor. The cell lysates were harvested and mixed with $2 \mu \mathrm{g}$ anti-hemagglutinin (HA; CW0092; Beijing ComWin Biotech Co., Ltd., Beijing, China) or anti- $v$-myc avian myelocytomatosis viral oncogene homolog (Myc; CW0088; Beijing ComWin Biotech Co., Ltd.) antibody and placed on a shaker for overnight at $4^{\circ} \mathrm{C}$. Subsequently, protein A/G agarose beads were added and then rocked at $4^{\circ} \mathrm{C}$ for $4 \mathrm{~h}$. The pulled-down proteins were separated by $12 \%$ sodium dodecyl sulfate-polyacrylamide gel electrophoresis (SDS-PAGE) and then transferred to polyvinylidene difluoride membranes. The membranes were blocked with dried skim milk for $2 \mathrm{~h}$ at room temperature and the blot was probed with Myc monoclonal antibody $(1: 1,000)$ overnight at $4^{\circ} \mathrm{C}$. The membranes were then incubated for $2 \mathrm{~h}$ at $4^{\circ} \mathrm{C}$ with horseradish peroxidase-conjugated goat anti-mouse antibody (1:1,000; CW0102; Beijing ComWin Biotech Co., Ltd.). Bands were visualized using an ECL Western Blotting Detection System (Shanghai Majorbio Bio-Pharm Technology Co., Ltd., Shanghai, China) and imaged (34).

Subcellular colocalization of FXR1P and CMAS. HEK293T and HeLa cells were maintained in DMEM medium supplemented with $10 \% \mathrm{FBS}, 100 \mathrm{U} / \mathrm{ml}$ penicillin, and $100 \mathrm{mg} / \mathrm{ml}$ streptomycin. At 70\% confluency, HEK293T and HeLa cells were plated in laser scanning confocal petri dish with basal serum- and antibiotic-free DMEM. The plasmids pEGFP-N1-FXR1 and pDsRed-Monomer-N1-CMAS were single-transfected and co-transfected into HEK293T cells and HeLa cells using Lipofectamine 2000 (35). After 5 h, the medium was then replaced with complete DMEM containing $10 \% \mathrm{FBS}, 100 \mathrm{U} / \mathrm{ml}$ penicillin, and $100 \mathrm{mg} / \mathrm{ml}$ streptomycin. After $48 \mathrm{~h}$, the transfected cells were collected and then washed twice with phosphate-buffered saline. Subsequently, $4 \%$ paraformaldehyde was used to fix the transfected cells and the nuclei were stained with 4',6-diamidino-2-phenylindole (Beyotime Institute of Biotechnology, Nantong, China) for $10 \mathrm{~min}$. A laser confocal microscope (Carl Zeiss AG, Oberkochen, Germany) was used to observe the localization and colocalization of CMAS and FXR1P in the cells.

Monosialotetrahexosylganglioside 1 (GM1) enzyme-linked immunosorbent assay (ELISA). HEK293T and SH-SY5Y cells were maintained in basal DMEM and DMEM/F12, respectively, lacking FBS and antibiotics. HEK293T and SH-SY5Y cells were divided into normal cell, empty vector and $F X R 1$ overexpression group cells. The empty vector group and $F X R 1$ overexpression group cells were transfected with the empty plasmid pcDNA3.1(-) and recombinant vector pcDNA3.1 (-)-FXR1, respectively, using Lipofectamine 2000 (36). Subsequently, cells from each group were collected and lysed. Lysates were centrifuged to remove cell debris ( $400 \times \mathrm{g}, 5 \mathrm{~min}, 4^{\circ} \mathrm{C}$ ), and ELISA was performed by a human anti-GM1 ELISA kit (YM-L0587; Shanghai YuanMu Biological Technology Co., Ltd., Shanghai, China) according to the manufacturer's protocol. Measurements in all groups were repeated six times.

Statistical analysis. Data are presented as the mean \pm standard deviation. The association among three groups was assessed by analysis of variance using SPSS software, version 19.0 (IBM SPSS, Armonk, NY, USA). P<0.05 was considered to indicate a statistically significant difference.

\section{Results}

FXR1P-interacting protein screening using the yeast two-hybrid system. The bait plasmid pGBKT7-FXR1 was constructed with complete human FXRI and the prey plasmids pGADT7-cDNA were constructed with the complete Human Fetal Brain Matchmaker cDNA library. The results of sequencing analysis demonstrated that the inserts were in-frame and the restriction sites were correct. Subsequently, AH109-pGBKT7-FXR1 with Y187-pGADT7-cDNA 
transformants were mated on SD/LTH medium and $\beta$-gal assays were performed. In total, 10 positive colonies were formed on the SD/LTH medium and exhibited positive results in the $\beta$-gal assay. Following sequencing and analysis, a colony that produced a 434-amino acid protein with high identity with human CMAS (99\% identity) was identified and used in the subsequent experiments.

Validation of the interaction between FXRIP and CMAS using the yeast two-hybrid system. To validate the interaction between the bait protein and prey protein from the cDNA library, Y187-pGADT7-cDNA colonies and AH109-pGBKT7-FXR1 were mated again and cultured on SD/LTH medium plates. Prey plasmid was recovered as described previously from colonies that continued to grow after 5 days on SD/LTH medium. Subsequently, the $\beta$-gal assay demonstrated a positive result. Additionally, no $\beta$-gal activity was observed in the yeast strain transformed with only the bait plasmid (pGBKT7-FXRl).

Directing verification of interaction between FXRIP and CMAS by co-immunoprecipitation. To verify the direct interaction between FXR1P and CMAS, two recombinant vectors, pCMV-HA-FXRl and pCMV-Myc-CMAS, were constructed and the results of sequencing analysis suggested that the inserts were in-frame and the restriction sites were correct. Subsequently, pCMV-Myc-CMAS was co-transfected with pCMV-HA-FXR1 into HEK293T cells, and cell extracts were immunoprecipitated using the polyclonal anti-HA antibody or anti-Myc antibody, and protein A/G agarose beads. Following precipitation with anti-HA antibody, CMAS-Myc was detected using anti-Myc antibody and FXR1P-HA using anti-HA antibody. Furthermore, following precipitation with the anti-Myc antibody, FXR1P-HA was detected by anti-HA antibody (Fig. 1) and CMAS-Myc was detected by anti-Myc antibody (Fig. 1). Additionally, a negative control was performed using IgG (Fig. 1). The results indicated that the demonstrated interaction between FXR1P and CMAS is not because of nonspecific binding with $\mathrm{IgG}$.

FXR1P colocalizes with CMAS in HEK293T and HeLa cells. To measure the intracellular interaction between FXR1P and CMAS, two recombinant eukaryotic expression vectors, pEGFP-N1-FXR1 and pDsRed-N1-CMAS, were constructed. pEGFP-N1-FXR1 contained a gene segment of FXR1 and pDsRed-N1-CMAS contained a gene segment of CMAS. All recombinant constructs were sequence-verified. HEK293T and HeLa cells were transfected with the constructs, and the expression and subcellular distribution of these proteins were analyzed by laser confocal microscopy.

HEK293T and HeLa cells transfected with pEGFP-N1-FXRI exhibited strong cytoplasmic fluorescence, whereas transfection with pDsRed-N1-CMAS produced fluorescence in the nucleus (Fig. 2). Thus, the fusions to the N-terminus of EGFP or DsRed did not affect the fluorescence properties of the native protein, allowing the fusion protein to be correctly localized in vivo.

To evaluate the colocalization of FXR1P and CMAS, pEGFP-N1-FXR1 and pDsRed-N1-CMAS plasmids were transiently cotransfected into HEK293T and HeLa cells (Fig. 3). Transfection with pEGFP-N1-FXR1 produced green fluorescence, predominantly in the cytoplasm, whereas pDsRed-N1-CMAS transfection predominantly produced red fluorescence in the nucleus and less in the cytoplasm. The two polypeptides (pEGFP-N1-FXR1 and pDsRed-N1-CMAS) maintained intracellular localization, with yellow fluorescence demonstrating FXR1P and CMAS colocalization in the cytoplasm and the edge of the nucleus (Fig. 3). Typically, these genes are expressed in different parts of the cell, with no fluorescence overlap, but co-overexpression leads to the formation of oligomers in the cytoplasmic and nucleus, producing yellow fluorescence.

Overexpression of FXR1 increases the concentration of GM1 in SH-SY5Y cells. The current study demonstrated that FXR1P interacts with CMAS. CMAS catalyzes the synthesis of CMP-sialic acid, which is an activated form of sialic and the raw material of ganglioside synthesis. Accordingly, CMAS activity has been demonstrated to be indirectly associated with amount of the sialic acid converted to GM1 in cells. To estimate the effect of FXR1P overexpression on CMAS activity, recombinant vector pcDNA (-) 3.1-FXRl was constructed and then the concentration of GM1 was measured in SH-SY5Y and HEK293T cells transfected with pcDNA3.1 (-)-FXR1. ELISAs demonstrated that the concentration of GM1 was significantly increased in SH-SY5Y cells transfected with recombinant vector compared with untransfected cells ( $\mathrm{P}=0.016$; Table II; Fig. 4A), whereas there was no significant difference between the empty vector-transfected SH-SY5Y cells compared with untransfected cells (Table II; Fig. 4A). However, there was no significant difference in GM1 concentration among the three groups of HEK293 cells (Fig. 4B). These results demonstrated that overexpression of FXR1 increases the concentration of GM1 in SH-SY5Y cells by promoting CMAS activity, but not in HEK293T cells (Table II; Fig. 4B).

\section{Discussion}

FMRP and FXR1P members of the FMR protein family. It has been previously demonstrated that FMRP is highly expressed in neurons (37) and involved in the development of dendritic spines, and the plasticity and structural remodeling of synapses $(38,39)$. Deficiency of FMRP can cause mental retardation and impaired memory and learning. It was previously demonstrated that FXR1P and FMRP are co-expressed in certain tissues and regulated other proteins at the translational level (40). A previous study demonstrated that when FXRl is expressed normally in the brain tissue of patients with FXS, there were no significant neuropathological abnormalities, which indicated that FXR1P may rescue the function of FMRP (41). Currently, it remains unclear whether the mechanism of FXS is associated with FXR1P, but a variety of protein and factors are likely to be involved in this process. Thus, the study of protein and factors that interact with FXR1P will aid the clarification of the function of FXR1P in the pathogenesis of FXS.

Various methods, including co-immunoprecipitation, the yeast two-hybrid system, surface plasmon resonance and pull-down technology, are used to investigate interactions between proteins. The current study screened proteins using the yeast two-hybrid system, and demonstrated that CMAS 


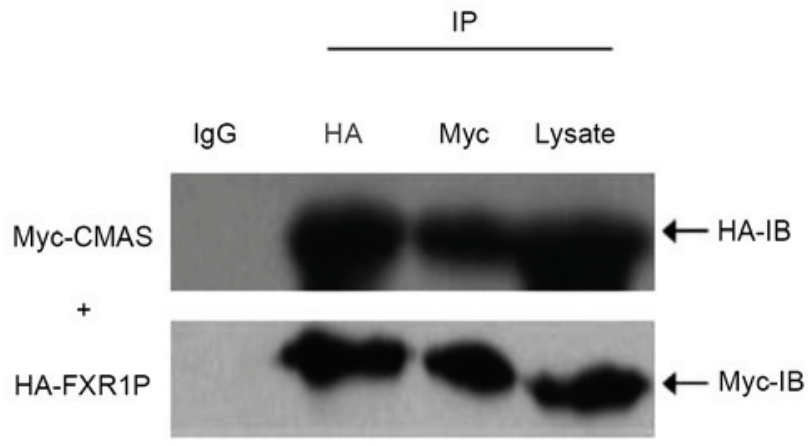

Figure 1. Co-IP of FXR1P and CMAS in HEK293T cells. Cells were transfected with pCMV-HA-FXR1 and pCMV-Myc-CNS, then lysates were subjected IP using anti-HA or anti-Myc mouse monoclonal antibodies, normal mouse IgG used as a negative control. The IP components of the assay and whole cell lysate were examined by western blot analysis with anti-HA and anti-Myc antibodies. IP, immunoprecipitation; IgG, immunoglobulin G; HA, hemagglutinin tag; Myc, $v$-myc avian myelocytomatosis viral oncogene homolog; CMAS, CMP-N-acetylneuraminic acid synthetase; FXR1P, fragile $X$ related 1 ; IB, immunoblot.

interacted with FXR1P. However, the two-hybrid system tends to identify a high proportion of false positives (protein hits that are unlikely to associate in vivo) (42). Thus, the results were validated using immunoprecipitation and cellular colocalization analysis in subsequent experiments.

Co-immunoprecipitation is a powerful and simple method to detect the interaction between proteins. Its principle is that certain binding proteins are preserved intact when cells are lysed under non-denaturing conditions. Based on the specificity between the antibody and the antigen, a protein of interest (protein X) can be immunoprecipitated with a targeted antibody, then protein $\mathrm{Y}$, which binds with protein $\mathrm{X}$, can be also precipitated. In the present study, the interaction between FXR1P and CMAS was investigated using this method. SDS-PAGE and western blotting demonstrated that CMAS was also precipitated when FXR1P was pulled-down by a specific antibody from the total proteins of cells co-transfected with pCMV-Myc-CMAS and pCMV-HA-FXRI (Fig. 1). This demonstrated an interaction between FXR1P and CMAS. However, co-immunoprecipitation does no fully to explain the specific interaction between two proteins in vivo, because the interaction may not be direct and may be an indirect interaction through other proteins (43). Thus, the result of co-immunoprecipitation was validated by colocalization analysis of CMAS and FXR1P cells. The areas where the proteins interact in cells were demonstrated by laser confocal microscopy.

For intracellular colocalization experiments, HEK-293T cells and HeLa cells were transfected with an EGFP-FXR1 fusion vector and DsRed-CMAS fusion vector. This demonstrated that FXR1P and CMAS were respectively located in the cytoplasm and nuclei. However, when the cells were co-transfected with both the fluorescent protein fusion vectors, the merge signal was observed in the cytoplasm and nucleus. The interaction between the two proteins was confirmed by co-immunoprecipitation in vitro, and the overlapping fluorescence signal indicated there was an interaction between FXR1P and CMAS in live mammalian cells.

CMAS is an enzyme that can catalyzes the synthesis of sialic acids, which are a family of nine-carbon sugars

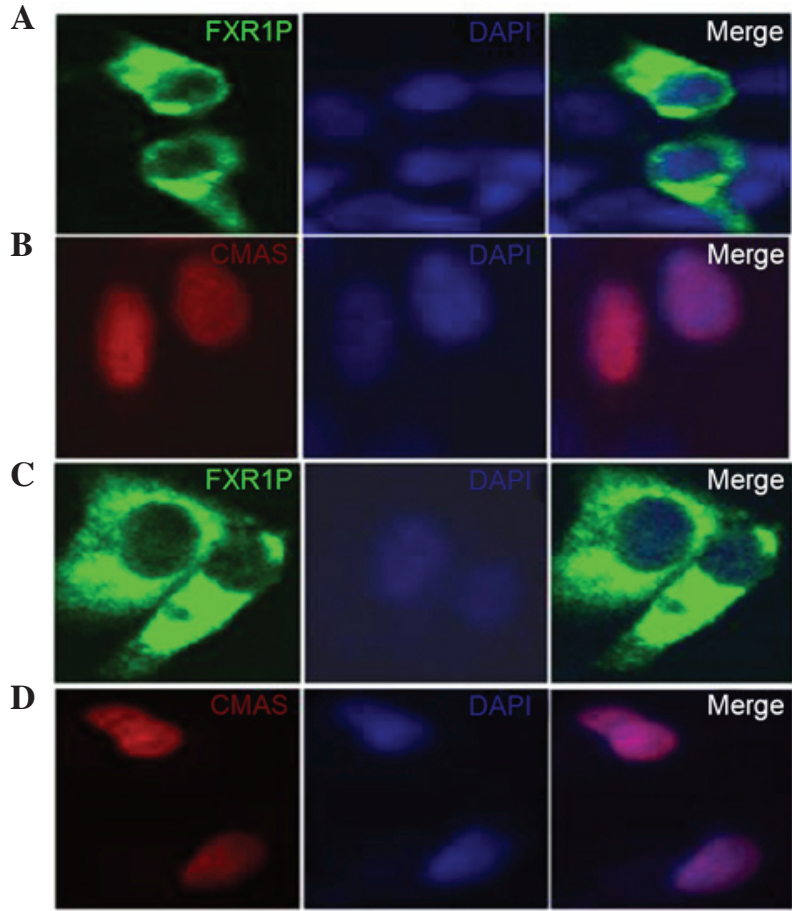

Figure 2. Expression and localization of FXR1P and CMAS in HEK293T cells and HeLa cells. HEK293T cells respectively transfected with (A) pEGFP-N1-FXR1 and (B) pDsRed-N1-CMAS FXR1P is located in the cytoplasm. CMAS is located in the nucleus. HeLa cells respectively transfected with (C) pEGFP-N1-FXR1 and (D) pDsRed-N1-CMAS. Cells were visualized by fluorescence microscopy. FXR1P is also located in the cytoplasm and CMAS in the nucleus. The nuclei were stained with DAPI. Magnification, x100. FXR1P, fragile X related 1; CMAS, CMP-N-acetylneuraminic acid synthetase; DAPI, 4',6-diamidino-2-phenylindole.

on cell surface glycoproteins and glycolipids important for determining the structure and function of various animal tissues, including cell-cell communications and immune responses (44). Sialylation occurs in two stages; activation of sialic acid by the enzyme CMAS and transfer to the target molecule to generate various GMs $(45,46)$. The current study demonstrated that overexpression of FXR1 increased the concentration of GM1 in SH-SY5Y cells, which may be caused by increased CMAS activity. However, this effect was limited to SH-SY5Y cells, and the GM1 concentration in HEK293T cells was not changed by FXR1 overexpression. It is well established that gangliosides promote the occurrence, growth and differentiation of nerve in the nervous system, however, HEK293T are human embryonic kidney cells. Thus, it is speculated that the regulation of GM1 level is tissue-specific.

Previous investigation has demonstrated that the level of sialic acid is very high in the brain and important for various cellular activities (47), including cell adhesion and migration, neurite growth, neuron differentiation and synapse formation. Additionally, it has been previously demonstrated that high levels of sialic acid increased the concentration of gangliosides and promoted the development of cognitive ability (48). For some patients with neurological diseases, including Alzheimer's disease and schizophrenia, the levels of GM1 is decreased in the blood and the brain $(49,50)$.

In conclusion, FXR1P may enhance the activation of sialic acid via interaction with CMAS, and increase the GM1 levels 
Table II. GM1 concentration in two cell lines under conditions of different expression level of FXRl.

\begin{tabular}{lccc}
\hline & Normal group & Empty vector group & Overexpression of FXR1 group \\
\hline SH-SY5Y & $13.938 \pm 0.126$ & $13.786 \pm 0.231$ & $18.245 \pm 0.241^{\mathrm{a}}$ \\
HEK293T & $10.213 \pm 0.084$ & $10.975 \pm 0.187$ & $12.138 \pm 0.382$ \\
\hline
\end{tabular}

${ }^{\text {aP }}<0.05$ vs. normal group, $\mathrm{n}=6$. GM1, monosialotetrahexosylganglioside; FXR1, fragile $\mathrm{X}$ related 1 .

A
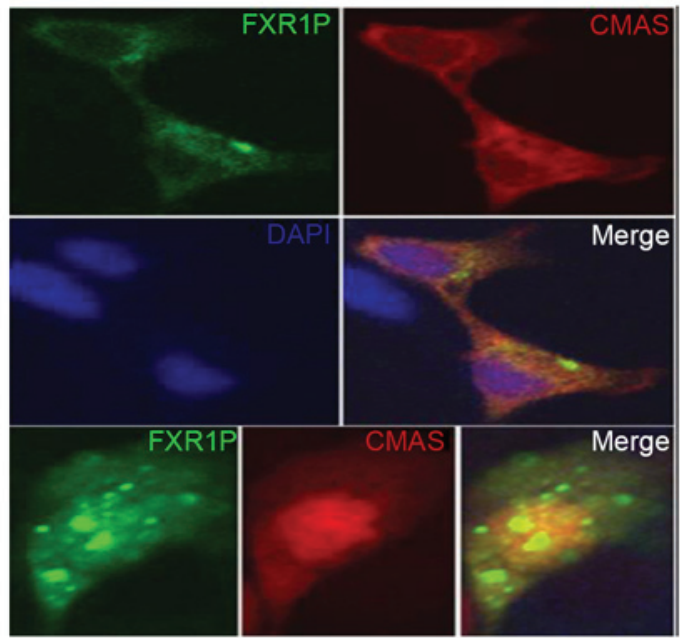

$\mathbf{B}$
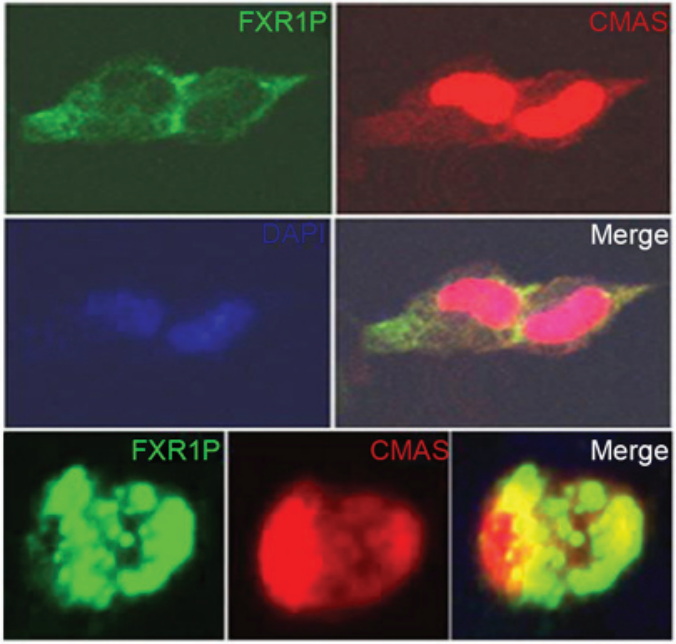

Figure 3. Colocalization of FXR1P and CMAS in HEK293T and HeLa cells. (A) HEK293T and (B) HeLa cells were transiently transfected with pEGFP-N1-FXR1 and pDsRed-N1-CMAS, then visualized by fluorescence microscopy. FXR1P (green) is visible in the cytoplasm, CMAS (red) is visible mostly in the nucleus, with less in the cytoplasm. Positive colocalization (yellow) is visible in the cytoplasm and around the nucleus. Yellow indicated the colocalization of pEGFP-FXR1P with pDsRed-CMAS. The nuclei (blue) were stained by DAPI. Magnification, x100. FXR1P, fragile X related 1; CMAS, CMP-N-acetylneuraminic acid synthetase; DAPI, 4',6-diamidino-2-phenylindole.

to affect the development of the nervous system. The results of the present study indicate that FXR1P interacts with CMAS in vivo, and that FXR1P and CMAS can co-localize in the cytoplasm around the nucleus. Additionally, FXR1P is able to enhance CMAS activity to increase the concentration of GM1 in SH-SY5Y cells. Taken wit the results of a previous
A

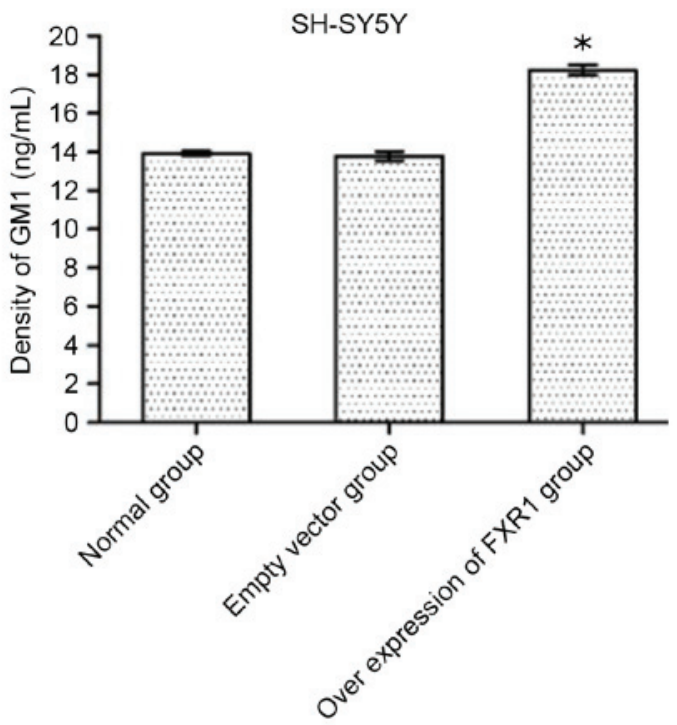

B

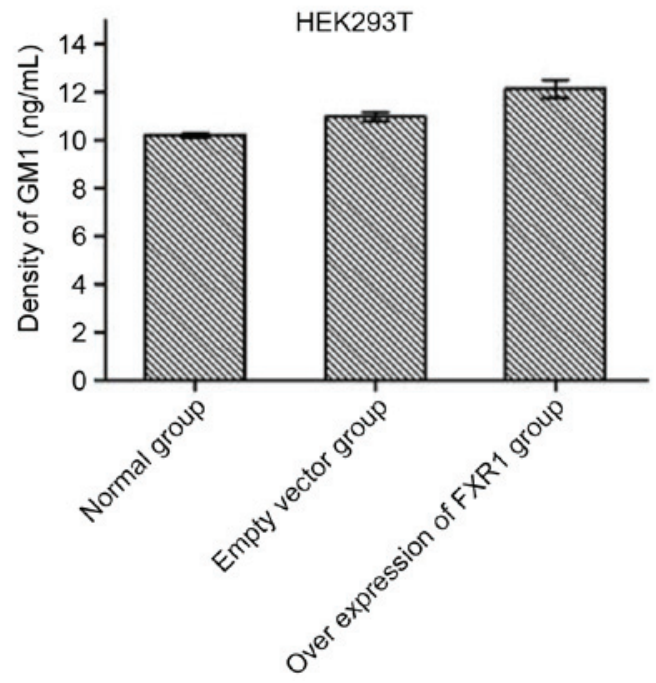

Figure 4. Effect of different expression levels of the FXR1 gene on the concentration of GM1. (A) SH-SY5Y cells transfected with pcDNA3.1(-)-FXR1, (B) HEK293T cells transfected with pcDNA3.1(-)-FXR1, then lysed and enzyme-linked immunosorbent assay was performed. The GM1 concentration was detected by ND 2000c trace UV spectrophotometer. In SH-SY5Y cells, the GM1 concentration was not significantly increased when comparing the empty vector group with normal group, but there was a significant increase in the FXR1 gene overexpression group compared with the normal group $(\mathrm{n}=6)$. ${ }^{\mathrm{P}} \mathrm{P}<0.05$ vs. normal group. There were no significant differences in GM1 concentration in HEK293T cells among all three groups $(P>0.05$, $\mathrm{n}=6$ ). GM1, monosialotetrahexosylganglioside; FXR1P, fragile X related 1 .

study (51), the present study suggests that FXR1P serves an important role in the development of the brain and nervous system. 


\section{Acknowledgements}

This research was supported by the National Natural Science Foundation of China (grant no. 81200881) and the Hunan Provincial Natural Science Foundation of China (grant no. 12JJ6073).

\section{References}

1. Vincent A, Heitz D, Petit C, Kretz C, Oberlé I and Mandel JL: Abnormal pattern detected in fragile-X patients by pulsed-field gel electrophoresis. Nature 349: 624-626, 1991.

2. Verkerk AJ, Pieretti M, Sutcliffe JS, Fu YH, Kuhl DP, Pizzuti A, Reiner O, Richards S, Victoria MF, Zhang FP, et al: Identification of a gene (FMR-1) containing a CGG repeat coincident with a breakpoint cluster region exhibiting length variation in fragile $\mathrm{X}$ syndrome. Cell 65: 905-914, 1991.

3. Kremer EJ, Pritchard M, Lynch M, Yu S, Holman K, Baker E, Warren ST, Schlessinger D, Sutherland GR and Richards RI: Mapping of DNA instability at the fragile $\mathrm{X}$ to a trinucleotide repeat sequence $\mathrm{p}(\mathrm{CCG}) \mathrm{n}$. Science 252: 1711-1714, 1991.

4. Siomi MC, Siomi H, Sauer WH, Srinivasan S, Nussbaum RL and Dreyfuss G: FXR1, an autosomal homolog of the fragile X mental retardation gene. EMBO J 14: 2401-2408, 1995.

5. Zhang Y, O'Connor JP, Siomi MC, Srinivasan S, Dutra A, Nussbaum RL and Dreyfuss G: The fragile X mental retardation syndrome protein interacts with novel homologs FXR1 and FXR2. EMBO J 14: 5358-5366, 1995.

6. Ashley CT Jr, Wilkinson KD, Reines D and Warren ST: FMR1 protein: Conserved RNP family domains and selective RNA binding. Science 262: 563-566, 1993.

7. Siomi H, Siomi MC, Nussbaum RL and Dreyfuss G: The protein product of the fragile $\mathrm{X}$ gene, FMR1, has characteristics of an RNA-binding protein. Cell 74: 291-298, 1993.

8. Brown V, Small K, Lakkis L, Feng Y, Gunter C, Wilkinson KD and Warren ST: Purified recombinant Fmrp exhibits selective RNA binding as anintrinsic property of the fragile X mental retardation protein. J Biol Chem 273: 15521-15527, 1998.

9. Darnell JC, Jensen KB, Jin P, Brown V, Warren ST and Darnell RB: Fragile X mental retardation protein targets $G$ quartet mRNAs important for neuronal function. Cell 107: 489-499, 2001.

10. Schaeffer C, Bardoni B, Mandel JL, Ehresmann B, Ehresmann C and Moine $\mathrm{H}$ : The fragile $\mathrm{X}$ mental retardation protein binds specifically to its mRNA via a purine quartet motif. EMBO J 20 : 4803-4813, 2001.

11. Eberhart DE, Malter HE, Feng Y and Warren ST: The fragile $\mathrm{X}$ mental retardation protein is a ribonucleoprotein containing both nuclear localization and nuclear export signals. Hum Mol Genet 5: 1083-1091, 1996.

12. Eberhart DE and Warren ST: The molecular basis of fragile $X$ syndrome. Cold Spring Harb Symp Quant Biol 61: 679-687, 1996

13. Winograd C and Ceman S: Fragile X family members have important and non-overlapping functions. Biomol Concepts 2: 343-352, 2011.

14. Siomi MC, Zhang Y, Siomi H and Dreyfuss G: Specific sequences in the fragile $\mathrm{X}$ syndrome protein FMR1 and the FXR proteins mediate their binding to $60 \mathrm{~S}$ ribosomal subunits and the interactions among them. Mol Cell Biol 16: 3825-3832, 1996.

15. Ceman S, Brown V and Warren ST: Isolation of an FMRP-associated messenger ribonucleoprotein particle and identification of nucleolin and the fragile X-related proteins as components of the complex. Mol Cell Biol 19: 7925-7932, 1999.

16. Tamanini F, Willemsen R, van Unen L, Bontekoe C, Galjaard H, Oostra BA and Hoogeveen AT: Differential expression of FMR1, FXR1 and FXR2 proteins in human brain and testis. Hum Mol Genet 6: 1315-1322, 1997.

17. Bakker CE, de Diego Otero Y, Bontekoe C, Raghoe P, Luteijn T, Hoogeveen AT, Oostra BA and Willemsen R: Immunocytochemical and biochemical characterization of FMRP, FXR1P, and FXR2P in the mouse. Exp Cell Res 258: $162-170,2000$.

18. Tamanini F, Van Unen L, Bakker C, Sacchi N, Galjaard H, Oostra BA and Hoogeveen AT: Oligomerization properties of fragile-X mental-retardation protein (FMRP) and the fragile-X-related proteins FXR1P and FXR2P. Biochem J 343: 517-523, 1999
19. Kirkpatrick LL, Mcllwain KA and Nelson DL: Alternative splicing in the murine and human FXR1 genes. Genomics 59: 193-202, 1999.

20. Mientjes EJ, Willemsen R, Kirkpatrick LL, Nieuwenhuizen IM, Hoogeveen-Westerveld M, Verweij M, Reis S, Bardoni B, Hoogeveen AT, Oostra BA and Nelson DL: Fxr1 knockout mice show a striated muscle phenotype: Implications for Fxrlp function in vivo. Hum Mol Genet 13: 1291-1302, 2004.

21. Khandjian EW, Bardoni B, Corbin F, Sittler A, Giroux S, Heitz D, Tremblay S, Pinset C, Montarras D, Rousseau F and Mandel J: Novel isoforms of the fragile $\mathrm{X}$ related protein FXR1P are expressed during myogenesis. Hum Mol Genet 7: 2121-2128, 1998.

22. Huot ME, Bisson N, Davidovic L, Mazroui R, Labelle Y, Moss T and Khandjian EW: The RNA-binding protein fragile $\mathrm{X}$-related 1 regulates somite formation in Xenopus laevis. Mol Biol Cell 16: 4350-4361, 2005.

23. Van't Padje S, Chaudhry B, Severijnen LA, van der Linde HC, Mientjes EJ, Oostra BA and Willemsen R: Reduction in fragile $\mathrm{X}$ related 1 protein causes cardiomyopathy and muscular dystrophy in zebrafish. J Exp Biol 212: 2564-2570, 2009.

24. Davidovic L, Sacconi S, Bechara EG, Delplace S, Allegra M, Desnuelle $\mathrm{C}$ and Bardoni B: Alteration of expression of muscle specific isoforms of the fragile $\mathrm{X}$ related protein 1 (FXR1P) in facioscapulohumeral muscular dystrophy patients. J Med Genet 45: 679-685, 2008.

25. Khera TK, Dick AD and Nicholson LB: Fragile X-related protein FXR1 controls post-transcriptional suppression of lipopolysaccharide-induced tumour necrosis factor-alpha production by transforming growth factor-beta1. FEBS J 277: 2754-2765, 2010.

26. Zarnescu DC and Gregorio CC: Fragile hearts: New insights into translational control in cardiac muscle. Trends Cardiovasc Med 23: 275-281, 2013.

27. Blech-Hermoni Y and Ladd AN: RNA binding proteins in the regulation of heart development. Int J Biochem Cell Biol 45: 2467-2478, 2013.

28. Ma Y, Wang C, Li B, Qin L, Su J, Yang M and He S: $\mathrm{Bcl}-2$-associated transcription factor 1 interacts with fragile X-related protein 1. Acta Biochim Biophys Sin (Shanghai) 46: 119-127, 2014.

29. Huang X, Qiu YL, Tan JY, Qiao YF and Li M: Effect of UBE2C overexpression on the proliferation of 293Tcell line. Xi Bao Yu Fen Zi Mian Yi Xue Za Zhi 27: 634-636, 2011 (In Chinese)

30. Chen Y, Sittler A, Yu M, Bardoni B and Wu G: Screening of proteins interact with FMR1 by yeast two-hybrid system. Zhongguo Yi Xue Ke Xue Yuan Xue Bao 20: 173-178, 1998 (In Chinese).

31. Mockli N and Auerbach D: Quantitative beta-galactosidase assay suitable for high-throughput applications in the yeast two-hybrid system. Biotechniques 36: 872-876, 2004.

32. Zou J, Mi L, Yu XF and Dong J: Interaction of 14-3-30 with KCMF1 suppresses the proliferation and colony formation of human colon cancer stem cells. World J Gastroenterol 19: 3770-3780, 2013.

33. Zheng Y, Zhang LP, Jia XQ and Wang HY: Construction of a yeast two-hybrid cDNA library from the human testis. Zhonghua Nan Ke Xue 18: 310-313, 2012 (In Chinese).

34. Ma Y, He S, Yang Y, Chen Q, Xiao W, Li B, Jiao S and Fu X: Ferritin, heavy polypeptide 1 interacts with fragile X-related protein 1. Neural Regen Res 6: 790-796, 2011.

35. Duan Z, Chen J, He L, Xu H, Li Q, Hu S and Liu X: Matrix protein of Newcastle disease virus interacts with avian nucleophosmin B23.1 in HEK293T cells. Wei Sheng Wu Xue Bao 53: 730-736, 2013 (In Chinese).

36. Yuan SF, Shi CH, Yan W, Yao Q, Li NL, Wang T, Wang L and Zhang YQ: Construction and expression of eukaryotic coexpression plasmid containing human MUC1 gene and GM-CSF gene. Xi Bao Yu Fen Zi Mian Yi Xue Za Zhi 23: 18-20, 24, 2007 (In Chinese).

37. Abitbol M, Menini C, Delezoide AL, Rhyner T, Vekemans M and Mallet J: Nucleus basalis magnocellularis and hippocampus are the major sites of FMR-1 expression in the human fetal brain. Nat Genet 4: 147-153, 1993.

38. Dictenberg JB, Swanger SA, Antar LN, Singer RH and Bassell GJ: A direct role for FMRP in activity-dependent dendritic mRNA transport links filopodial-spine morphogenesis to fragile X syndrome. Dev Cell 14: 926-939, 2008. 
39. Bassell GJ and Warren ST: Fragile X syndrome: Loss of local mRNA regulation alters synaptic development and function. Neuron 60: 201-214, 2008.

40. Cook D, Sanchez-Carbente Mdel R, Lachance C, Radzioch D, Tremblay S, Khandjian EW, DesGroseillers L and Murai KK. Fragile $\mathrm{X}$ related protein 1 clusters with ribosomes and messenger RNAs at a subset of dendritic spines in the mouse hippocampus. PLoS One 6: e26120, 2011.

41. Agulhon C, Blanchet P, Kobetz A, Marchant D, Faucon N, Sarda P, Moraine C, Sittler A, Biancalana V, Malafosse A and Abitbol M: Expression of FMR1, FXR1, and FXR2 genes in human prenatal tissues. J Neuropathol Exp Neurol 58: 867-880, 1999.

42. Guo D, Rajamäki ML and Valkonen J: Protein-protein interactions: The yeast two-hybrid system. Methods Mol Biol 451: 421-439, 2008.

43. Monti M, Orrù S, Pagnozzi D and Pucci P: Interaction proteomic. Biosci Rep 25: 45-56, 2005.

44. Horsfall LE, Nelson A and Berry A: Identification and characterization of important residues in the catalytic mechanism of CMP-Neu5Ac synthetase from Neisseria meningitides. FEBS J 277: 2779-2790, 2010

45. Qasba PK, Ramakrishnan B and Boeggeman E: Substrate-induced conformational changes in glycosyltransferases. Trends Biochem Sci 30: 53-62, 2005.
46. Samuels NM, Gibson BW and Miller SM: Investigation of the kinetic mechanism of cytidine 5'-monophosphate N-acetylneuraminic acid synthetase from Haemophilus ducreyi with new insights on rate-limiting steps from product inhibition analysis. Biochemistry 38: 6195-6203, 1999.

47. Sánchez-Felipe L, Villar E and Muñoz-Barroso I: $\alpha 2-3$ - and

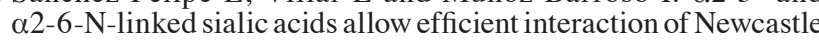
Disease Virus with target cells. Glycoconj J 29: 539-549, 2012.

48. Gurnida DA, Rowan AM, Idjradinata P, Muchtadi D and Sekarwana N: Association of complex lipids containing gangliosides with cognitive development of 6-month-old infants. Early Hum Dev 88: 595-601, 2012.

49. Hirano-Sakamaki W, Sugiyama E, Hayasaka T, Ravid R, Setou M and Taki T: Alzheimer's disease is associated with disordered localization of ganglioside GM1 molecular species in the human dentate gyrus. FEBS Lett 589:3611-3616, 2015

50. Sommer BR, Cohen BM, Satlin A, Cole JO, Jandorf L and Dorsey F: Changes in tardive dyskinesia symptoms in elderly patients treated with ganglioside GM1 or placebo.J Geriatr Psychiatry Neurol $7: 234-237,1994$.

51. Ma Y, Qin L, Don X, Li B, Wang C, Xu C,Wang S and He S: Biological effect of the interaction between mental retardation related protein (FXR1P) and CMAS. Prog Biochem Biophys 40: 1124-1131, 2013. 